\title{
Kernos
}

Revue internationale et pluridisciplinaire de religion grecque antique

3| 1990

Varia

\section{Parole de poète, parole de prophète : les oracles et la mantique chez Pindare}

\section{Emilio Suárez de la Torre}

\section{(2) OpenEdition}

\section{Journals}

\section{Édition électronique}

URL : http://journals.openedition.org/kernos/1006

DOI : 10.4000/kernos.1006

ISSN : 2034-7871

\section{Éditeur}

Centre international d'étude de la religion grecque antique

\section{Édition imprimée}

Date de publication : 1 janvier 1990

ISSN : 0776-3824

\section{Référence électronique}

Emilio Suárez de la Torre, «Parole de poète, parole de prophète : les oracles et la mantique chez Pindare », Kernos [En ligne], $3 \mid$ 1990, mis en ligne le 19 avril 2011, consulté le 19 avril 2019. URL http://journals.openedition.org/kernos/1006 ; DOI : 10.4000/kernos.1006 


\section{PAROLE DE POÈTE, PAROLE DE PROPHÈTE : LES ORACLES ET LA MANTIQUE CHEZ PINDARE}

1. «Rends tes oracles, ô Muse, et je serai ton prophète !». «...je te supplie, ô Pytho la dorée... reçois l'interprète fameux des Piérides» ${ }^{1}$. Malgré les problèmes d'interprétation qu'ont posés ces vers, je crois qu'on ne peut pas douter des liens, je dirais même de l'identité, que notre poète établit entre les pouvoirs prophétiques et poétiques. C'est un fait qu'on a observé à maintes reprises, sous des points de vue plus ou moins divers : dans les scholies, chez les commentateurs modernes, etc., on trouve çà et là des remarques à cet égard. Des philologues tels que von Wilamowitz ${ }^{2}$ ont mis en relief les rapports entre Pindare et Delphes. Certains ouvrages traitent même, directement ou indirectement, de ce sujet $^{3}$. Faut-il ajouter que la terre natale de Pindare était le siège de plusieurs centres oraculaires extrêmement importants, comme celui d'Apollon Ismenios, ou que, dans la ville de Thèbes, Amphiaraos et son fils Alcméon avaient des sanctuaires oraculaires ${ }^{4}$ ? Néanmoins, à mon avis, il manquait une étude compréhensive tant de la présence de la mantique et des oracles dans la poésie de Pindare que (et surtout) du rôle qu'y jouent ces éléments. Je veux vous présenter aujourd'hui un résumé

1 Traduction d'A. PUECH, Pindare, vol. IV, Isthmiques. Fragments, fr. 32 et Péan 6, v. 3-6 respectivement. Pour les citations du texte grec, je suivrai l'édition de B. Snell-H. Maehler, Pindarus I. Epinicia, Leipzig, 19806; II. Fragmenta. Indices, Leipzig, $1975^{4}$.

2 Pindaros, Berlin, 1922 (reprint Zürich, Hildesheim, 1985), p. 66 sq.

3 Par exemple, G. NEBel, Pindar und die Delphik, Stuttgart, 1961; J. DEFradas, Pindare, poète delphique, in $I L, 21$ (1969), p. 127-34, et, en général, dans toutes les études qui ont envisagé l'oracle de Delphes et aussi la divination en Grèce (cf. P. Roth, Mantis : The Nature, Function and Status of a Greek Prophetic Type, Diss. [Bryn Mawr, Pennsylvania, 1982] Ann Arbor, 1986, passim). D'ailleurs, malgré la coïncidence partielle dans le titre, on sait que l'œuvre de J. Duchemin, Pindare, poète et prophète, Paris, 1955, n'étudie que très accidentellement les aspects oraculaires et, pour ainsi dire, strictement prophétiques du poète.

4 Cf. S. Symeonoglou, The Oracles of Thebes, in La Béotie Antique (Lyon Saint-Étienne, 1983), Paris, 1985, p. 155-58, avec des données archéologiques récentes et, dans le présent volume, la contribution de P. BONNECHÈRE, Les oracles béotiens, p. 53-65, sur l'évolution des oracles locaux. 
des données fournies par la révision à laquelle j'ai soumis les textes du poète béotien et les conclusions auxquelles j'ai été amené concernant la fonction et la signification de ces aspects prophétiques et divinatoires ${ }^{5}$.

2. Il faut donc se demander quels sont les moyens que le poète utilise pour souligner son status et pour conférer à ses compositions ces traits prophétiques. Et, en même temps, essayer de démontrer que de la présence de ces éléments, on peut tirer des conséquences qui justifient le titre de cette communication. Je le ferai d'après la classification qui suit :

2.1. La présence de devins et d'autres personnages mythiques ou des dieux en fonction prophétique en est le premier moyen. Parmi les premiers, on peut citer Amphiaraos ${ }^{6}$, Cassandre $^{7}$, Iamos $^{8}$, Mélampos ${ }^{9}$, Mopsos ${ }^{10}$, Polyide ${ }^{11}$, Ténéros ${ }^{12}$, Tirésias ${ }^{13}$; à ceux-ci, il faut ajouter quelques références à des manties des sanctuaires apolliniens mentionnés d'une façon collective ${ }^{14}$. L'autre groupe, très important, est celui des héros tels qu'Héraclès ${ }^{15}$, Médée ${ }^{16}$ ou le Centaure Chiron ${ }^{17}$, et aussi la déesse Thémis ${ }^{18}$, qui exercent des fonctions semblables dans les épisodes dont ils sont les protagonistes. Ils partagent des traits très importants qui surpassent même l'art divinatoire (ce qui, d'ailleurs, est

5 Pour une exposition détaillée des données et de mon interprétation, vid. mon étude Adivinación y profecía en Píndaro I, in Minerva, 2 (1988), p. 65-106; II, Minerva, 3 (1989 sous presse).

$6 \quad O .6,16 \mathrm{~b}-17 ;$ P. 8,38 sq.; N. 9, 16 sq.; 10, 8-9; I. 7, 33; fr. 43 (dub.). Sur les particularités du culte oraculaire des héros, cf. A. BRELICH, Gli eroi greci. Un problema storico-religioso, Roma, 1958, p. 106-113 ("L'eroe e la mantica»); et sur les aspects thérapeutiques, ibidem, p. 113-118 («L'eroe e la iatrica»).

$7 \quad P .8,20$ sq. et, peut-être, Péan 8 a (fr. 52i).

$8 \quad$ O. 6, 45 sq. et passim.

9 P. 4, 126; Péan 4 (fr. 52d) 28 sq.

10 P. 4, 194 et 201.

$110.13,65 \mathrm{sq}$.

12 Hymnes, fr. 51d; Péan 7 (fr. 52g) 13; 9 (fr. 52k) 41-2.

13 N. 1,61 sq.

14 Péan 8 (fr. 52i); fr. 192 et 202.

15 I. $6,41 \mathrm{sq}$.

$16 P .4,41$ sq. et passim.

$17 P .3,1$ et passim; 4, 103 et $115 ; 6,19-27 ; 9,29$ sq.; N. 3, 53; 4, 60; I. 8, 41; fr. 177c.

18 I. $8,31 \mathrm{sq}$. 
caractéristique de ces figures mythiques $)^{19}$. Tout d'abord, quelques-uns sont des fils nés des "amours d'Apollon" ${ }^{20}$ (Iamos, Ténéros), dieu qui leur a octroyé ces dons. En général, les sources nous parlent très souvent de leurs pouvoirs médico-magiques (Iamos, Polyide, Chiron, Médée); mais ils dominent les techniques les plus diverses : l'ornithomancie. (Iamos, Mélampos, Mopsos, Héraclès), l'empyromancie (Iamos), les sortes ou l'interprétation des prodiges les plus divers (Mopsos), l'onirocrisie (Polyide), etc. De toutes ces puissances, Pindare en choisit et en met en relief seulement quelques-unes (on pourrait dire "d'après les besoins du scénario") et il en ajoute d'autres que je considère assez importantes du point de vue symbolique. Par exemple, nous pouvons trouver Amphiaraos en fonction de conseiller de son fils Amphiloque, auquel il propose de suivre la «norme du Polype» 21 . Chiron est aussi un modèle de prudence et de sagesse, l'éducateur par excellence, auquel on a recours dans des circonstances délicates ${ }^{22}$ et dont le conseil est respecté même par les dieux ${ }^{23}$. Ténéros, fils d'Apollon et de Mélie, est décrit comme "prophète des themites" 24 du dieu, ce qui nous fait penser au terme themistopólos, approprié aux rois et aux juges. Nous sommes donc devant une figure qui est très proche du roi-prêtre archaïque ${ }^{25}$ et qui est le médiateur entre les dieux et la communauté. Cette définition est aussi celle que reçoivent les manties d'Apollon à Delphes ${ }^{26}$. De plus, il faut souligner le fait qu'Amphiaraos rend sa prophétie sur la victoire des Épigones et le destin d'Adraste ( $P, 8,44$ sq.) depuis l'au-delà puisque, d'après la définition de M. Detienne, il est (avec Tirésias) un de ces hommes «qui sont des 'vivants' dans le monde des morts, qui sont pourvus d'une mémoire dans le monde de l'oubli» 27.

19 Il faut ajouter que, à côté de ces personnages proprement prophétiques (y compris aussi Alcméon, $P$. 8, 46 et 57 sq.), on sait que Pindare en mentionna d'autres qui possédaient des traits chamaniques, tels Abaris (fr. 270) et Aristéas (fr, 271).

20 C'est le titre d'un article d'A.A. STEFos, Les amours d'Apollon dans l'œuvre de Pindare, in Platon, 27 (1975), p. 162-181; vid. du même auteur, Apollon dans Pindare (Thèse, Paris, 1973), Athènes, 1975.

21 Cf. les remarques de B. GENTILI dans le chapitre «Poeta-committente-pubblico" de son ouvrage Poesia e pubblico nella Grecia antica, Roma, 1984, p. 153-202.

22 I. 8, 41, à propos des noces de Pélée et Thétis.

23 Ainsi Apollon dans la Pythique 9.

24 Péan 9 (fr. 52k), 41-42.

25 Cf. RoTH, op cit., p. 124 et 144, n. 1.

26 Fr. 192.

27 Les maîtres de vérité dans la Grèce archaïque, Paris, $1981^{2}$, p. 74, avec n. 140. 
2.2. La prophétie et son langage est le deuxième procédé. J'ai recueilli une vingtaine d'oracles ou de prophéties directement ou indirectement cités par le poète. La plupart d'entre eux ${ }^{28}$ appartiennent au dieu oraculaire par excellence, Apollon (14); un seul à Hécate ${ }^{29}$, et cinq autres sont des prophéties prononcées par les devins ou héros en fonction mantique ${ }^{30}$. D'autres dieux ou sanctuaires oraculaires étaient mentionnés dans l'ceuvre de Pindare (par exemple le Zeus de Dodone ou Zeus Ammon) ${ }^{31}$, mais l'état des textes ne nous confirme pas la présence d'oracles.

Pindare montre une grande habileté pour harmoniser le langage consacré par la tradition prophétique et le langage poétique ${ }^{32}$. Les traits oraculaires que j'ai observés appartiennent à l'un de ces trois groupes :

28 Les destinataires sont: Cadmos (fr. $32=142$ Parke-Wormell); le roi Épytos $(O .6,37-8$ et 47-9 = 141 PW); Erginos $(P .8,100-11)$; Agamèdes et Trophonios (fr. 2-3, 2); Laìos (fr. $68=148$ PW); Pélias (P. 4, 71 sq. = 143 PW et $P .4,159$ sq. = 144 PW); Éaque (O. 8, 42-6); Panthoos (Péan 6, fr. 56f, 71 sq.); Paris-Alexandre (Péan 8a, fr. 52i, 14-25); Battos (P. 4, 4 sq. = 39 PW et passim; cf. $P .5,55$ sq.); les Spartiates $(I .7,12-15=146 \mathrm{PW})$; Tlépolème $(O .7,31-4)$.

29 Péan 2 (fr. 52b), 73-9.

30 Cf. la liste supra, 2.1, avec les notes.

31 Dodone : fr. 59; Zeus Ammon : P. 4, 16; fr. 36, 58.

32 Marie DeLCOURT s'étonnait de l'opinion des éditeurs à propos de l'oracle de Crésus et la tortue 'au chaudron' (HDT., I, 471) et ses ressemblances avec les vers 43-49 de la Pythique 9, car ils affirmaient que Pindare imitait la langue et les formules des oracles. Elle proposait quant à elle l'hypothèse inverse, qu'elle trouvait «infiniment plus vraisemblable» (L'oracle de Delphes, Paris, 1955, p. 107). Aucun des auteurs qui ont étudié la langue des oracles n'a écouté l'avertissement de Mme DELCOURT (cf., par exemple, W.E. McLEOD, Oral Bards at Delphi, in TAPhA, 92 (1961), p. 317-25; L.E. RossI, Gli oracoli comme documento d'improvisazione, in M. CANTILENA, C.O. PAVESE (éd.), I Poemi epici rapsodici non-omerici e la tradizione orale, Atti del Convegno di Venezia 1977, Padova, 1981, p. 203-230). On a même proposé l'existence d'une tradition de poésie oraculaire continentale très ancienne, indépendante de la tradition épique (J. A. Fernández Delgado, Poesía oral mántica en los oráculos de Delfos, in J.L. Melena (éd.), Symbolae L. Mitxelena septuagenario oblatae, Vitoria, 1985, p. 153-66; Los oráculos y Hesíodo. Poesía oral mántica y gnómica griega, Cáceres, 1987; cf. les précisions de J.Mํ. NiETo IвÁÑ̄Ez, Fórmulas homéricas y lenguaje oracular, in Minerva, 2 (1988), p. 33-46. Je crois qu'il ne faut pas aller si loin ni dans un sens ni dans l'autre : Pindare a pu connaître une tradition de vers oraculaire assez ancienne (en tout cas bénéficiaire de, et influencée par, la tradition épique) qui se servait de certains procédés poétiques considérés comme plus appropriés à la nature des réponses divines. Mais ceci n'exclut pas la possibilité que les 'compositions' plus tardives aient profité de la 
a) Les termes «techniques» qui, à l'intérieur des oracles, nous rappellent que l'accomplissement de ces faits était prédestiné ${ }^{33}$.

b) Des formules ou expressions formulaires qui organisent habituellement la structure de la réponse oraculaire ${ }^{34}$.

c) D'autres traits stylistiques des réponses (communs en bonne mesure avec les caractéristiques de la langue poétique en général) accumulés dans les oracles inclus dans les compositions pindariques. C'est l'ensemble le plus représentatif de l'harmonisation du langage oraculaire avec l'expression poétique ${ }^{35}$.

2.3. La structure et l'organisation interne des odes est le troisième moyen (et le plus complexe). La présence de tous les éléments énumérés précédemment n'est pas due au hasard et n'obéit pas seulement à des exigences momentanées du récit ou du cours des idées. Par contre, nous allons voir qu'elle répond à un but fonctionnel évident, avec des conséquences parfois très importantes pour la structure de la composition et, du point de vue du contenu, elle est inséparable de l'intentionnalité du poète de faire refléter dans ses compositions la conception qu'il a de son métier.

Je vais me borner maintenant aux Épinicies. En réalité le panorama des autres compositions fragmentaires n'est pas très différent et des chants «apolliniens» par nature, comme les Péans, ne font que renforcer ce qu'on peut déduire des chants de victoire ${ }^{36}$.

langue des poètes non épiques, y compris (et au premier rang, bien sûr) le grand poète delphique, Pindare.

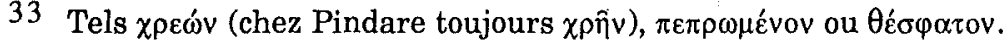

34 Cf., par exemple, les caractéristiques de la prophétie de Tirésias (N. 1, 61 sq.) où il ne manque pas même la "condition précédente" (d'après la formule de J. FonTENROSE, The Delphic Oracle. Its Responses and Operations, with a Catalogue of Responses, Berkeley-Los Angeles, 1978 [19812], p. 179); un exemple d'adaptation syntaxique se trouve dans la $P .4,7$ (style indirect) et on a aussi des formules transformées en périphrase expressive $(P .4,75)$.

35 Des remarques sur ce sujet déjà chez F. DoRnserff, Pindars Stil, Berlin, 1928, p. 40-41. La plupart d'entre elles sont fondées sur l'ambiguïté des réponses oraculaires, à laquelle on peut arriver par des moyens divers : tel, par exemple, le 'kenning' (cf. $O .13,80-1$ ); ou même l'ambiguïté syntaxique, car c'est ainsi qu'à mon avis peuvent être expliquées les difficultés des vers 73-75 du Péan 2.

36 À l'appui de cette affirmation peuvent être apportés, par exemple, les

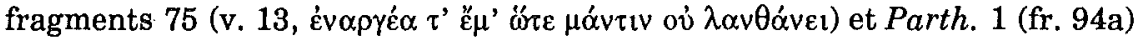

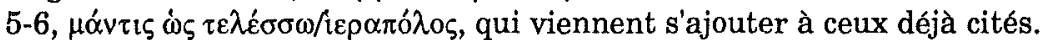


Il faut mettre en vedette la Pythique $4^{37}$. Les prophéties y créent des espaces narratifs et redistribuent les séquences temporelles. La composition «en anneau» (observée très souvent par les commentateurs) est fondamentale dans tout le poème, mais avec la particularité qu'elle repose surtout sur les passages oraculaires. Nous sommes donc en face d'une sorte de 'canevas' prophétique, dont l'ensemble est encadré par les oracles d'Apollon à Battos (4 sq., 53 sq., 259 sq.) en alternance avec les références à l'actualité ( 1 sq., 64 sq., 263 sq. et, plus concrètement, 270 sq.). Enfermé dans ce premier niveau se trouve un deuxième encadrement structurel qui a comme points de repère la prophétie sur l'île de Théra (13 sq.) et l'évocation de son accomplissement (258). Ch. Segal a remarqué que les particularités de ces séquences chronologiques traduisent pour les participants au kômos «la perspective des choses éternelles" ${ }^{38}$. Certainement; et j'ajouterai que de cette façon le poète ne met pas seulement en relief la victoire chantée, mais aussi sa propre fonction, son rôle de médiateur dans tout ce processus.

Envisageons maintenant la Pythique 9. L'identité poète-prophète est ici très importante et est réussie par des moyens assez complexes. Par exemple, par les connotations de certains personnages (Chiron, Aristée $^{39}$, Nélée ${ }^{40}$ ) et surtout grâce à ce noyau du poème qui est la réponse de Chiron à Apollon (39 sq.). Le parallèle Chiron-poète, Apollonvainqueur ${ }^{41}$ émerge d'un réseau de liaisons entre les plans mythique et actuel. Ainsi Chiron sanctionne de sa parole infaillible ce qu'accomplira tout de suite Apollon : Pindare confirme et révèle la victoire de Télésicrate, assimilée de cette façon à la soumission d'une

37 Ch. SEGal, Pindar's Mythmaking : the Fourth Pythian Ode, Princeton, 1986, a mis en relief le rôle de la prophétie, dans cette ode. En ce qui concerne le 'temps' prophétique et poétique, cf. A. Hurst, Temps du récit chez Pindare (Pyth, 4) et Bacchylide (11), in $M H, 40$ (1983), p. 154-168.

38 Op cit., p. 51.

39 Sur quelques traits intéressants de ce personnage, cf. M. DETIENNE, Orphée au miel, in QUCC, 12 (1971), p. 7-23.

40 Un autre 'maître de vérité'; cf. M. DeTienne, Les Maîtres... (op. cit.), p. 39 sq.

41 Pour une exposition des arguments qui m'ont conduit à cette interprétation, $\mathrm{cf}$. mon article El mito de Cirene y la victoria de Telesicrates (Pind. Pyth. IX), in Apophoreta Philologica Emmanueli Fdez. Galiano Oblata, I (= EClás 87), Madrid, 1984, p. 199-208, avec bibliographie sur l'ode (vid. n. 2-3), à laquelle on ajoutera : J. PòrTulas, Lectura de Pindar, Barcelona, 1977, p. 78-98; A. KöHNKEN, 'Meilichos orga'. Liebesthematik und aktueller Sieg in der neunten Pythischen Ode Pindars, in Pindare, Vandœuvres-Genève, 1985 (Entretiens sur l'Antiquité classique, 31), p. 71-111, ainsi que les remarques de Ch. SEGAL, op. cit., p. 165 sq. 
vierge sauvage ( $a$ pattern très important dans la mythologie grecque); Chiron prédit la naissance d'un fils d'Apollon et de Cyrène doué des qualités exceptionnelles de ses parents ${ }^{42}$ : Pindare chante la prouesse de l'homme qui habite dans la ville de l'héroïne, une victoire conquise sur la terre qui est le siège par excellence de la parole prophétique (v. 75), etc.

La structure de cette Pythique a aussi des similitudes avec celle de la Quatrième. Par exemple, l'introduction du premier paradigme mythique (Apollon-Cyrène) coïncide avec un «écart chronologique» 43 qui nous amène en ascendant jusqu'au niveau le plus lointain. Au vers 70 , un premier cercle est fermé ${ }^{44}$. Mais ça se fait en deux séquences, l'une condensée $(5 \mathrm{sq}$.$) , l'autre analytique (26 \mathrm{sq} .)^{45}$.

Prophétique par excellence est sans doute l'Olympique 6, dédiée à un vainqueur apparenté aux Iamides, les descendants du fils d'Apollon et d'Évadne consacrés au service de l'autel mantique de Zeus à Olympie. Encore une fois les aspects prophétiques et "amoureux" d'Apollon vont de pair. Depuis le commencement (avec l'image architectonique, qui fait penser à un palais, mais aussi à un temple) il y a un assemblage

42 Le climax établi par le contenu de la prophétie, jusqu'à l'annonce de la naissance d'Aristée, a déjà été souligné par L. ILLIG, Zur Form der pindarischen Erzählung. Interpretation und Untersuchungen, Borna-Leipzig, 1931, p. 45 : «Die prophetische Rede gipfelt in dem visionär eschauten Bild der Gottwerdung des Sohnes (59-65)».

43 L'expression a été empruntée à A. Hurst, Aspects du temps chez Pindare, in Pindare, Vandœuvres-Genève, 1985 (Entretiens sur l'Antiquité classique, 31), p. 156-197 (vid. p. 160).

44 D'ailleurs, ce premier mythe propose un modèle pour les deux autres (celui qui met en scène Iolaos, v. 79 sq., et les noces des Danaïdes et d'Alexidame avec la 45 fille d'Antée, v. 106 sq.).

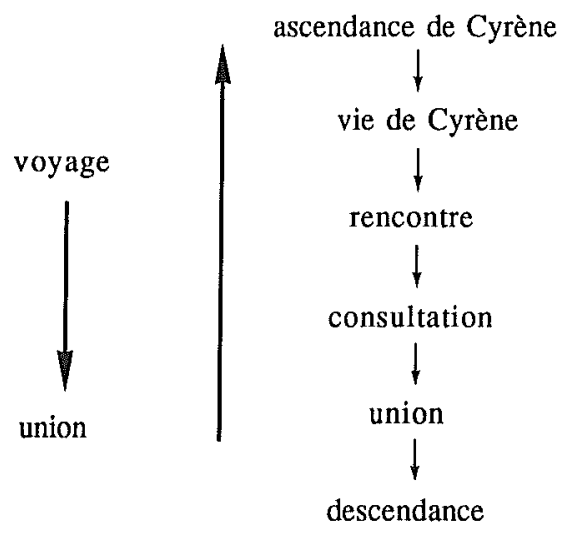


parfait des plans thématiques (prophétie, poésie, victoire sportive) et chronologiques (actualité, passé mythique, projection future de la parole poétique-prophétique). On constate l'importance de la liaison (qu'elle soit explicite ou suggérée) chant-parole d'un côté, et des personnages et des faits en rapport avec la prophétie d'un autre ${ }^{46}$. L'approche poèteIamos est vraiment remarquable ${ }^{47}$.

C'est le mérite d'A. Hurst d'avoir saisi la signification profondément prophétique qu'enferme l'Olympique 7. Celle-ci est caractérisée par «une certaine fréquence des remontées le long de l'axe chronologique» 48 , avec trois points de repère : le poète $(20 \mathrm{sq.})$, l'oracle d'Apollon (32 sq.) et les «légendes des hommes» (54 sq.); à tel point que «les procédés de récit du dieu sont ainsi présentés comme parallèles de ceux du poète» 49 . Du point de vue structural, on aperçoit une disposition en "cercles concentriques" qui encadrent ces mouvements de régression-retour ${ }^{50}$. Des réseaux conceptuels sont aussi à souligner : par exemple, ceux formés par les termes lógos (et synonymes) - phrênsophía et les oppositions autour des significations «droit»/«détourné» (ce

46 On pourrait parler, par exemple, d'un parallèle Adraste-Pindare : on nous dit même que son éloge d'Amphiaraos est prononcé à Thèbes (v. 16). Il y a une sorte d'affirmation prophétique-patriotique. Il ne faut pas oublier que dans la Thébaïde Adraste recevait l'épithète de $\mu \mathrm{\varepsilon} \lambda$ ínnpus (cf. Plat., Phèdre, 269a),

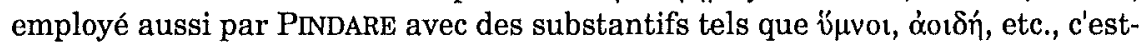
à-dire, dit de ses chants. En outre, pour le symbolisme de l'abeille, des couleurs, etc. cf. DUChemin, op. cit., p. 251 sq.; les connotations chthoniennes du personnage d'Iamos et leurs conséquences pour les pouvoirs du poète) ont été remarquées par J. STERN, The myth of Pindar's 'Olympian' 6, in AJPh, 91 (1970), p. 332-340 (vid.p. 338-340)

47 STERN (loc. cit.) avait affirmé déjà que «ce qui est vrai d'Iamos en des termes mythiques l'est également de la poésie de Pindare».

48 Op. cit., en n. 43, p. 172.

49 Ibid.

50

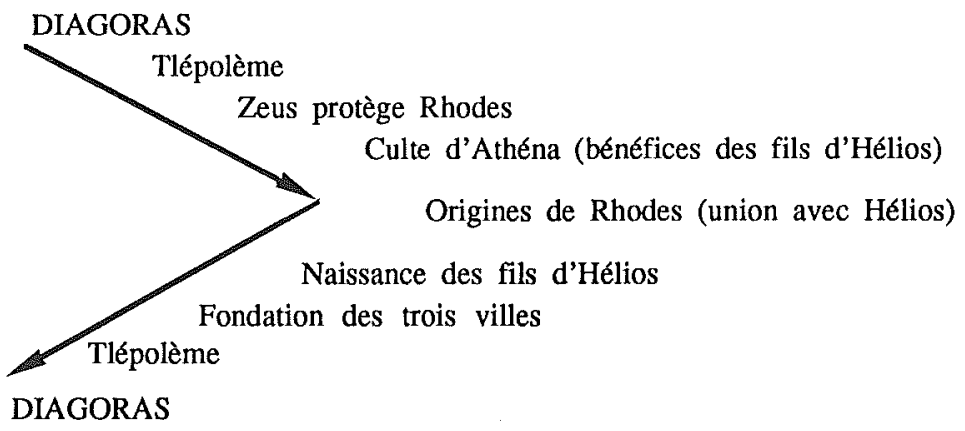


qui signifie souvent "vrai»/(faux»), sans oublier le couple "occultation»/«révélation» 51 .

La Néméenne 1 a une structure très particulière (c'est une des «variantes uniques» selon la terminologie de R. Hamilton ${ }^{52}$ ) qui laisse le mythe pour la seconde moitié de l'ensemble, en un climax parfait à partir du proème jusqu'à la prophétie finale ${ }^{53}$. Je pense que le rapport action prodigieuse (d'Héraclès), prophétie; victoire, épinicie (et, en conséquence, mantis, poète) est ici très net. Il est souligné par une distribution du contenu ajustée aux quatre triades. Cette harmonieuse disposition (selon la structure habituelle en anneau) aboutit à l'expression des bons vœux du poète envers la communauté du vainqueur, en parfaite correspondance avec la première triade, ce qui laisse entrevoir ce message de la part du poète : la fortune actuelle et les triomphes continueront à l'avenir.

En dehors de ces odes, la fonction des prophéties dans le reste des épinicies n'est pas sans valeur. L'Isthmique 8, par exemple, nous montre très bien ce qu'on pourrait appeler «l'enchaînement diachronique des effets de la parole prophétique et de la parole poétique». L'intervention de Thémis ( $31 \mathrm{sq}$.) arrête une querelle fatale et en donne l'orientation droite et juste. Son fruit ne peut pas périr (45-6), tout comme la parole du poète chante et immortalise ceux qui sont déjà partis. À son tour Pindare, mêlant la parole prophétique et l'action mythique, non seulement glorifie le moment présent victorieux, mais fait nourrir aussi l'agatha elpis (15 a) qu'on doit conserver pour opérer la traversée de la vie ${ }^{54}$.

51 En rapport avec les odes déjà commentées on reconnaît le modèle suivant pour le mythe choisi : a) Union du dieu avec une femme (- île ou ville : ici, HéliosRhodes); b) Garantie du serment (parole de vérité); et c) (cf. $P .9$ ) descendance des éponymes mythiques du territoire colonisé plus tard par les ancêtres du vainqueur. Le récit de ces faits est construit sur le schéma : faute (ou défaut) oracle - fondation (cf. A. BREsson, Deux légendes rhodiennes, in Les grandes figures religieuses. Fonctionnement pratique et symbolique dans l'Antiquité [Besançon 1984], Paris, 1986, p. 411-421).

52 Epinikion. General Form on the Odes of Pindar, The Hague-Paris, 1974.

53 ILLIG, op. cit., p. 25; cf. aussi les remarques de D.E. GERBER, What Time can do (Pindar, Nemean 1, 46-7), in TAPhA, 93 (1962), p. 30-33 et Ch. SEgAL, Time and the Hero : the Myth of Nemean 1, in $R h M, 117$ (1974), p. 29-39, surtout en ce qui concerne les rapports temps-prophéties; J.K.-F.S. NEWMaN, Pindar's Art. Its Traditions and Aims, Hildesheim, 1984, parlent de la «valeur télescopique et de la prophétie" au sujet de cette ode (p. 69, 96, etc.).

54 Cf. l'introduction à cette Isthmique de G.A.Privitera, Pindaro. Le Istmiche, Milano, 1982, p. 120-121, ainsi que les notes ad loc. Contre le fait de rattacher 
Dès son commencement, la Pythique 3 établit un schéma "concentration-expansion» assez remarquable ${ }^{55}$. Tout entière, elle devient une exemplification du mêdèn ágan delphique, matérialisé par le va-et-vient des exemples mythiques : la conduite insensée de Coronis, la convoitise d'Asclépios, la folie des filles de Cadmos et d'Harmonie. Mais sur ce fond rejaillit d'une lumière plus intense la fortune réelle du laudandus ainsi que la puissance restauratrice et curative du poète ${ }^{56}$. La force de la parole poétique ferme la composition : elle transforme en perdurable (chronía, v. 115) la vertu, elle est parole véritable.

L'Olympique 8 a une "ouverture prophétique» (1-7): Olympie est "mère de la vérité», le lieu où les vertus personnelles et de famille sont démontrées par le triomphe sportif (après avoir été confirmées par la parole mantique); après, elles seront proclamées et immortalisées par le chant de victoire. Le pouvoir immortalisant de la parole poétique ferme aussi l'ode, car le poète nous explique comment seront annoncés ces triomphes aux ancêtres morts ${ }^{57}$ (et il exprime ses vœux pour l'avenir). Mais le centre de la composition est la prophétie prononcée par Apollon sur la conquête de Pergame, en interprétant ainsi le prodige des serpents (42 sq.). Le dieu annonce et confirme un avenir glorieux pour les descendants d'Éaque. La réplique au niveau de l'actualité du chant n'est pas très loin : après un appel à la promatheia (60), l'éloge de l'aleiptes semble recouvrir un parallèle avec la figure du poète. Les fonctions de l'entraîneur exemplifient la «prévision» puisqu'il montre

cette ode aux événements historiques s'est exprimé D.S. CARNE-ROss, Pindar, New Haven-London, 1985, p. 121 sq. Sur les particularités pindariques du récit mythique, cf. Th.K. HuBBaRD, Two notes on the myth of Aeacus in Pindar, in GRBS, 28 (1987), p. 5-22. Les relations métis-thémis-oracle et les différences entre cette ode et le Prométhée enchaîné ont été étudiées récemment par M. CoRsano, Themis. La norma e l'oracolo nella Grecia antica, Galatina (Lecce), 1988.

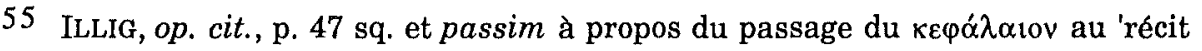
libre' du mythe; M. LefkowITz, The Victory Ode, Parke Ridge, N.J., 1976, p. $142 \mathrm{sq}$.

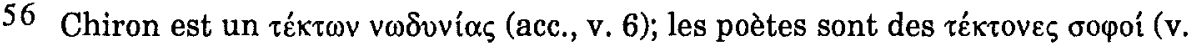
113). Sur les aspects 'thérapeutiques' de l'épinice on consultera la contribution de R.P. MARTIN au Premier Congrès International de mythologie et Psychothérapie (Angoisse et Divination), tenu à Paris-Delphes en 1988, intitulée La poésie de Pindare et la thérapie de la parole : le poete guérisseur par le mythe (sous presse). Je dois cette référence à la gentillesse du Prof. F. Jouan.

57 Cf. T. Nishimura, Dead relatives in Epinician Odes, in JCS, 33 (1985), p. 1-8 (résumé en anglais, p. 133-134); Ch. SEGAL, Messages to the Underworld. An aspect of poetic immortalization in Pindar, in AJPh, 106 (1985), p. 199-212. 
"la méthode et les pratiques efficaces pour entraîner l'homme destiné à remporter aux Jeux sacrés la gloire tant désirée» (Puech) ${ }^{58}$.

Enfin, la Pythique 8 construit sa louange du vainqueur, un enfant d'Égine, sur le fondement des mots du devin Amphiaraos adressés à Adraste et qui commencent par cette sentence : "La nature fait briller chez les enfants l'esprit généreux qu'ils tiennent de leurs pères» (44-5, tr. Puech). L'ode entière est présidée par les pouvoirs d'Apollon, positifs ou destructeurs, matérialisés par la techna (34) du poète, reflet des machanai du héros-prophète (Alcméon, v. 60). Cette techna est ici orientée à faire exceller le bonheur et la bonne fortune du vainqueur, ce qui s'accomplit par la voie des antithèses, le positif l'emportant toujours sur le négatif, de la même façon que le chant est une victoire sur le silence $^{59}$. Au reste, le contraste était déjà la caractéristique même de la prophétie d'Amphiaraos, qui avait prédit non seulement la victoire d'Alcméon et des Épigones, mais aussi la mort du fils d'Adraste.

\section{Conclusions}

3.1. En ce qui concerne le poète et la signification de son rôle, on peut parler d'une identité avec les devins et les prophètes. Tous les deux, poète et prophète, sont protégés par Apollon, qui leur a octroyé ces pouvoirs. De la même façon que le mantis interprète des signes, le poète en fait autant avec les signes de la victoire : il explique les raisons qui l'ont rendue possible et prédit des succès pour l'avenir. Ainsi, comme les anciens devins sont des hommes avec des pouvoirs prodigieux, le poète adoucit la rigueur des épreuves grâce à la force de la louange : sa parole a même des pouvoirs curatifs.

58 Bien entendu, le poète se situe à un niveau très supérieur : il peut aussi chanter la gloire de l'aleiptes !

59 Cette ode est l'illustration parfaite des oppositions établies par M. DETIENNE (op. cit., p. 25, mais pas à propos de cette ode) avec quelques additions. On pourrait les schématiser de la façon suivante :

\begin{tabular}{l|l} 
Positif & Négatif \\
mesure & démesure \\
tranquillité & inquiétude \\
supérieur & inférieur \\
lumière & ombre \\
CHANT & SILENCE \\
gloire & honte \\
vérité & mensonge \\
justice & violence \\
admiration & jalousie
\end{tabular}


3.2. Très étroits sont les liens entre la parole poétique et la parole prophétique. Celle-ci s'accomplit inéluctablement et, au moment d'être prononcée, elle matérialise ce qui s'annonce, elle devient réalité : elle ouvre un délai limité qui aboutira sans remède à l'accomplissement. Quant à la parole prophétique "poétisée», on peut parler d'une fonctionnalité immédiate et, simultanément, d'une signification dénotative plus large, qui laisse entrevoir une conception sacrée et du poète et de la poésie. Dans les compositions où sont présents les oracles, ceux-ci jouent un rôle décisif, car ils sont parfois les piliers qui supportent la structure de l'ode (par sa possibilité d'ouvrir les espaces du récit) ou bien ils sont contrebalancés par les expressions du poète sur le vainqueur et le destin de sa famille. Pour ces mêmes raisons, les conséquences dépassent le cadre momentané d'une ode concrète : la parole du poète, comme celle du prophète, est en dernier lieu celle d'Apollon, c'est une parole de vérité, qui ne connaît point le mensonge (et, ainsi, elle offre des garanties). Le poète, intermédiaire entre les hommes et les dieux, a aussi le pouvoir de changer la perspective chronologique ordinaire, de se placer (et de placer les événements) audelà du temps ou hors du temps. Devin et poète peuvent ranger, dans un tout susceptible d'être réordonné, "le présent, l'avenir et le passé». Plusieurs moyens contribuent à cette organisation de l'espace-temps poétique (polarisation, des échos verbaux, etc.) et à matérialiser dans chaque composition ces liens entre poésie et prophétie. Poète et prophète sont aussi à mi-chemin entre la vie et la mort : les voix des héros oraculaires arrivent de l'au-delà et, en sens contraire, les chants du poète annoncent les triomphes aux morts. Sans limites dans l'espace, la parole du poète atteint tous les coins de la Terre; sans limites dans le temps, ayant brisé l'oubli silencieux, elle immortalise à la fois sa gloire et celle du vainqueur.

Departamento de Filología Clásica

Emilio SUÁREZ DE LA TORRE

Facultad de Filosofia y Letras

Universidad de Valladolid

E - 47002 VALLADOLID 